\title{
Integrative modules for efficient genome engineering in yeast
}

\author{
Triana Amen ${ }^{1, *}$ and Daniel Kaganovich ${ }^{1, *}$ \\ ${ }^{1}$ Department of Cell and Developmental Biology, Hebrew University of Jerusalem, Givat Ram, Jerusalem 91904, Israel. \\ * Corresponding Authors: \\ Daniel Kaganovich, E-mail: dan@cc.huji.ac.il; \\ Triana Amen, E-mail: amentri@gmail.com
}

\begin{abstract}
We present a set of vectors containing integrative modules for efficient genome integration into the commonly used selection marker loci of the yeast Saccharomyces cerevisiae. A fragment for genome integration is generated via PCR with a unique set of short primers and integrated into HIS3, URA3, ADE2, and TRP1 loci. The desired level of expression can be achieved by using constitutive (TEF1p, GPD1p), inducible (CUP1p, GAL1/10p), and daughter-specific (DSE4p) promoters available in the modules. The reduced size of the integrative module compared to conventional integrative plasmids allows efficient integration of multiple fragments. We demonstrate the efficiency of this tool by simultaneously tagging markers of the nucleus, vacuole, actin, and peroxisomes with genomically integrated fluorophores. Improved integration of our new pDK plasmid series allows stable introduction of several genes and can be used for multi-color imaging. New bidirectional promoters (TEF1p-GPD1p, TEF1p-CUP1p, and TEF1p-DSE4p) allow tractable metabolic engineering.
\end{abstract}

doi: 10.15698/mic2017.06.576

Received originally: 05.01.2017; in revised form: 23.05.2017,

Accepted 24.05.2017,

Published 05.06.2017.

Keywords: vector, bidirectional promoter, integrative plasmid, genetic integration, yeast, Saccharomyces cerevisiae.

\section{Abbreviations:}

MCS - multiple cloning site; VHL - von Hippel Landau tumor suppressor.

\section{INTRODUCTION}

Saccharomyces cerevisiae is an indispensable tool for high throughput studies of biological processes. The everincreasing diversity of tools for yeast genome manipulation allows systematic examination of biological pathways in a highly physiological, cost-effective, and tractable manner [1-7]. However, attempts to introduce multiple genes into chromosomal loci often encounter complications due to decreased efficiency and cloning limitations, such as overlaps in restriction site usage amongst multiple inserts $[7,8]$. In order to overcome the difficulties of multiple gene integrations we sought to expand the variety of yeast integration cassettes with a specially designed pDK vector set.

Several methods enable rapid gene introduction. The most common ones are ectopic plasmid expression and chromosomal integration. Ectopic expression via multicopy or centromeric plasmids is often faster and easier than integration, but poses problems due to highly inhomogeneous expression $[9,10]$. Hence, multi-color imaging and metabolic engineering in yeast often require stable integration of genes. Genome integration via homologous recombination has advantages over ectopic expression: e. g. stable strains, controlled copy number, and uniform expression [9].

Several integration strategies are commonly employed. (1) Yeast integrative plasmids are introduced into the genome in linearized form [1]. The linearized fragment contains the desired insert and selection marker, but also substantial superfluous genetic material including the bacterial selection marker and replication origins [1]. These extraneous sequences may reduce integration efficiency [8]. Additionally, the customized insert must not contain the restriction site used for plasmid linearization, adding further limitations. (2) Another frequently used strategy is PCR amplification using extended primers with homology regions allowing integration into any locus. Since the primers provide relatively short homology regions, the integration efficiency is comparatively low with this approach as well [8]. Insertion into loci rather than selective markers does not pose a particular advantage since the selective marker locus still has to be present in the integrated fragment. (3) Recent advances in CRISPR/Cas9 genome engineering allow highly efficient introduction of multiple fragments with short homology regions and often without the need for a selective marker, however the strain must 
TABLE 1. pDK plasmid series with integrative modules.

\begin{tabular}{lllll}
\hline \multicolumn{1}{c}{ Module } & & & Locus \\
& HIS3 & URA3 & TRP1 & ADE2 \\
\hline TEF1p-MCS-TEF1t & pDK-HT & pDK-UT & pDK-TT & pDK-AT \\
\hline CUP1p-MCS-TEF1t & pDK-HC & pDK-UC & pDK-TC & pDK-AC \\
\hline $\begin{array}{l}\text { GAL1p-MCS(1)-CYC1t } \\
\text { GAL10p-MCS(2)-ADHt }\end{array}$ & pDK-HGG & pDK-UG & pDK-TG & pDK-AG \\
\hline $\begin{array}{l}\text { TEF1p-MCS(1)-CYC1t } \\
\text { GPD1p-MCS(2)-ADHt }\end{array}$ & pDK-HTG & pDK-UTG & pDK-TTG & pDK-ATG \\
\hline $\begin{array}{l}\text { TEF1p-MCS(1)-CYC1t } \\
\text { CUP1p-MCS(2)-ADHt }\end{array}$ & pDK-HTC & pDK-UTC & pDK-TTC & pDK-ATC \\
\hline $\begin{array}{l}\text { TEF1p -MCS(1)-CYC1t } \\
\text { DSE4p-MCS(2)-ADHt }\end{array}$ & pDK-HTD & pDK-UTD & pDK-TTD & pDK-ATD \\
\hline
\end{tabular}

also carry Cas9 and gRNA expressing cassettes [4, 11, 12]. (4) I-Scel-assisted integration has a significantly increased efficiency, but this approach also requires the additional expression of the meganuclease I-Scel locus [13]. (5) Plasmids carrying integrative cassettes excised by restriction with extended homology regions flanking the insert significantly improve integration efficiency $[6,9,14]$. We were inspired by this last strategy and decided to create a set of yeast integration cassettes that contain extended homology regions corresponding to a common selective marker locus, which eliminates the need to have a marker locus inside the cassette. An advantage of not having a selective marker locus inside the cassette reduces its size and potentially increases integration efficiency [8]. To reduce multiple integration time and to maximize available markers we constructed novel bidirectional promoter sets (Figure 1A).

The pDK series includes 24 plasmids which carry an integrative module for 4 common genetic markers (HIS3, URA3, ADE2, and TRP1). Constitutive (TEF1p, GPD1p), daughter specific (DSE4p) and inducible (CUP1p and bidirectional $G A L 1 / 10 p$ ) promoters flank multiple cloning sites. We also include 4 bidirectional promoters (GAL1p-GAL10p, TEF1p-GPD1p, TEF1p-CUP1p, and TEF1p-DSE4p) which allow multiple integrations (Figure 1, Table 1). PCR is carried out with short primers specific to a selectable marker in order to integrate the module (see Supplemental Table 1). The pDK set can be used for multi-color imaging, metabolic engineering, or any set of experiments that require stable genomic integration of one or more genes.

\section{RESULTS AND DISCUSSION \\ Vector overview}

We designed 24 pDK plasmids for stable expression of multiple genes in the yeast $S$. cerevisiae (Figure 1, Table 1). Vectors can be used for stable integration into 4 common $S$. cerevisiae selective marker loci HIS3, URA3, ADE2, and $T R P 1$. Plasmid variations include 6 promoters (Figure $1 C$ ): Constitutive TEF1 $p$, inducible CUP1 $p$, inducible bidirectional GAL1-10p in opposing orientation, constitutive bidirec- tional TEF1-GPD1p, constitutive-inducible bidirectional TEF1-CUP1p, constitutive-daughter-specific bidirectional TEF1-DSE4p (bidirectional promoter sets have two multiple cloning sites (MCS) in opposing orientation). The markers are split into two parts (see plasmid construction for details) and are flanking the region containing a promoter, MCS, and a terminator (Figure $1 \mathrm{~A}$ ). The fragment is amplified with primers specific to the marker (Supplemental Table 1). The orientation of the split markers allows integration of amplified region in a traditional homologous recombination driven way, resulting in doubling of the marker region (Figure 1B).

\section{Integrative modules allow stable and efficient integration of multiple inserts}

pDK vectors were examined for integration efficiency, correct integration, and stability of the insert. 24 integrative modules of PDK series were transformed into the W303 strain. PDK integrative modules have comparable integration efficiencies (Supplemental Figure 1A). Although double integration is possible we recommend sequential integration and using bidirectional promoters to expedite the work flow. We also compared the integration efficiency to other integration strategies: (1) pRS series - conventional linearized integrative plasmids [1], (2) EasyClone strategy extended homology regions, excisable marker and restriction based integration [6], and (3) extended primers $45 \mathrm{bp}$ homology region. In general, extended homology region based strategies provide higher efficiency of integration. $\mathrm{pDK}$ series integration is more effective than $\mathrm{pRS}$ series, and is comparable with EasyClone efficiency, which also has a reduced insert size but relies on restriction based integration.

To evaluate integration stability, a GFP reporter was cloned into the pDK series. The stability of the integration was tested by avoiding selection for more than 80 generations and then counting fluorescent cells (Supplemental Figure 1B). Modules are integrated with 95 - 100\% accuracy into the marker region as verified by PCR (Supplemental 
A

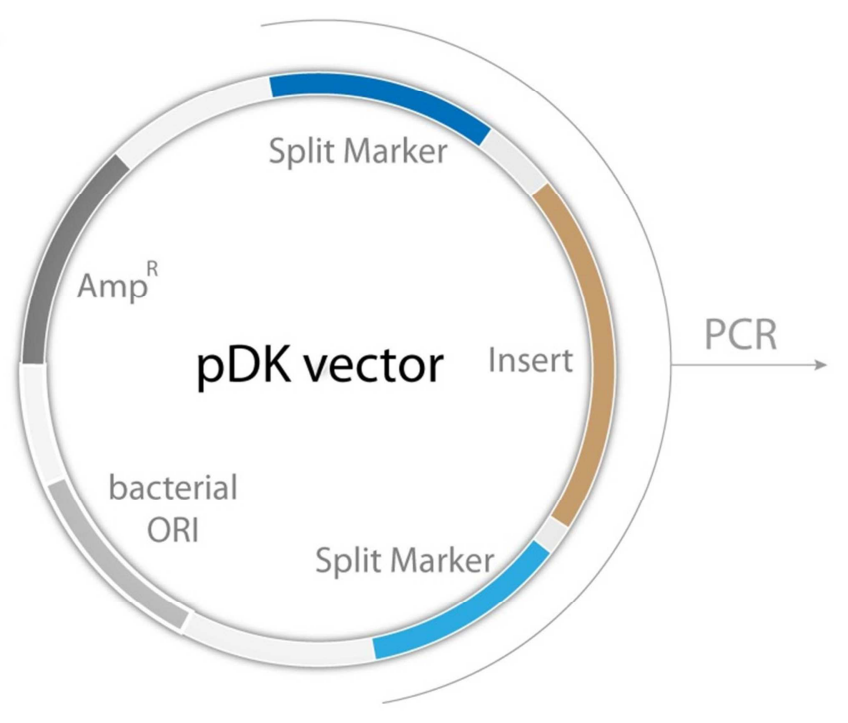

Integrative Module

\begin{tabular}{|c|c|c|c|c|c|}
\hline \multicolumn{2}{|c|}{ Split Marker } & \multicolumn{2}{|c|}{ Insert } & \multicolumn{2}{|c|}{ Split Marker } \\
\hline$A D E 2$ & & \multirow{4}{*}{\multicolumn{2}{|c|}{$\begin{array}{c}\text { Expression: } \\
\text { Inducible } \\
\text { Constitutive } \\
\text { Daughter-specific } \\
\text { Combinations }\end{array}$}} & \multirow{4}{*}{\multicolumn{2}{|c|}{$\begin{array}{l}\text { ADE2 } \\
\text { HIS3 } \\
\text { URA3 } \\
\text { TRP1 }\end{array}$}} \\
\hline HIS3 & & & & & \\
\hline URA3 & & & & & \\
\hline TRP1 & & & & & \\
\hline \multirow[t]{3}{*}{ Terminator 2} & \multirow[t]{3}{*}{ MCS } & \multirow[t]{3}{*}{ Promoter 2} & Promoter 1 & MCS & Terminator 1 \\
\hline & & & TEF1p & MCS & TEF1t \\
\hline & & & CUP1p & MCS & TEF1t \\
\hline$A D H t$ & MCS & GAL10p & GAL1p & MCS & $\mathrm{CYCt}$ \\
\hline$A D H t$ & MCS & GPD1p & TEF1p & MCS & $C Y C t$ \\
\hline$A D H t$ & MCS & CUP1p & TEF1p & MCS & $C Y C t$ \\
\hline$A D H t$ & MCS & DSE4p & TEF1p & MCS & $\mathrm{CYCt}$ \\
\hline
\end{tabular}

B

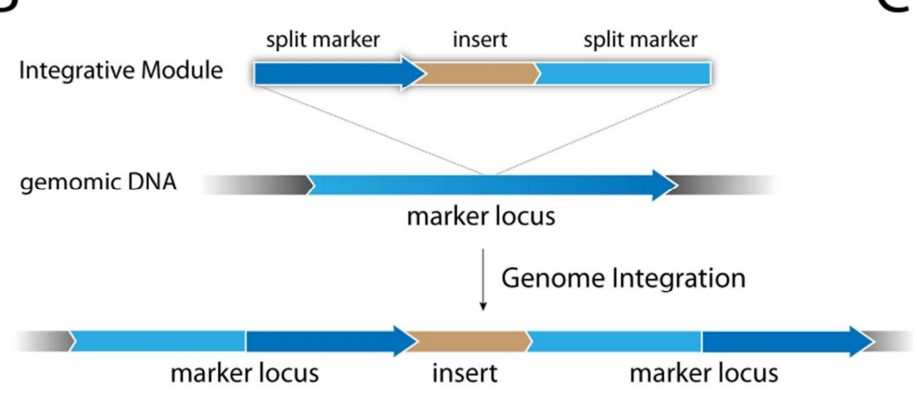

\begin{tabular}{|c|c|c|c|c|}
\hline promoter marker & HIS3 & URA3 & TRP1 & $A D E 2$ \\
\hline TEF1p & pDK-HT & pDK-UT & pDK-TT & pDK-AT \\
\hline CUP1p & pDK-HC & pDK-UC & pDK-TC & PDK-AC \\
\hline GAL1-GAL10p & pDK-HGG & pDK-UGG & pDK-TGG & pDK-AGG \\
\hline TEF1-GPD1p & pDK-HTG & pDK-UTG & pDK-TTG & PDK-ATG \\
\hline TEF1-CUPp & pDK-HTC & pDK-UTC & pDK-TTC & PDK-ATC \\
\hline TEF1-DSE4p & pDK-HTD & pDK-UTD & pDK-TTD & pDK-ATD \\
\hline
\end{tabular}

FIGURE 1: pDK vector series overview. (A) pDK vector with an integrative module flanked by a split marker. The customized insert is flanked by constitutive, inducible, or daughter-specific promoters. PCR is required for genetic integration. (B) Integration overview: the module is transformed into $S$. cerevisiae and inserted into the chromosome resulting in a marker locus duplication. (C) pDK vector set: constitutive, inducible, and four bi-directional promoter plasmids available for integration into four markers.

Figure 1B). Marker based integration is advantageous because it provides accurate targeting compared to the CRISPR/Cas9 strategy [15]. We also tested the stability of multiple integrations, since many of them carry same promoters and/or terminators, which can potentially lead to a loop-out of the fragments. Single and multiple integrations provide reliable homogenous expression levels under nonselective conditions (Supplemental Figure 1B, C).

\section{Application of pDK plasmids to multi-color imaging re- veals order of organelle inheritance}

As proof of concept for multi-color imaging we constructed peroxisomal, nuclear, and actin [16] markers using the pDK vector series and used the strain for 3D time-lapse microscopy (4D imaging) (Figure 2) [17]. Nuclear localization signal fused to a far-red fluorophore was used to visualize the nucleus. Peroxisome localization signal - tripeptide SKL was fused to the mCherry C-terminus to visualize peroxisomes. We also used LifeAct fused to GFP to visualize actin in living yeast [16]. The vacuolar marker $V P H 1$ was endogenously tagged with mBFP or GFP. Peroxisomes are known to use actin cables for inheritance [18]. Inp2, an integral peroxisomal membrane protein, binds the Myo2 motor ensuring localization to the bud $[19,20]$. Most of the organelles in yeast use actin cables for bud trafficking during division [21]. To determine whether organelle inheritance proceeds in a parallel or a serial way we examined the inheritance of the vacuole, peroxisomes, and the nucleus (Figure 2, Supplemental movie "wtdivision"). The nucleus itself is inherited in a microtubule dependent manner, however the astral microtubules are delivered on actin to the bud [22]. Multi-color imaging revealed that the first instance of peroxisome inheritance happens in parallel with the vacuole in the first $15 \mathrm{~min}$ of cell division (Figure 2B). Deletion of INP2 abolishes peroxisome inheritance, which has no effect on the time of vacuole inheritance [19] (Figure 2C, Supplemental movie "inpdivision"). Interestingly, the nucleus is inherited prior to the end of cell division. The integrative vector series that we have developed allows for efficient integration of up to 8 markers, enabling us to image cellular compartments simultaneously. 
A

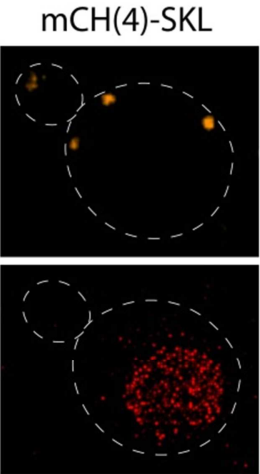

Vph1-mBFP
LifeAct-GFP
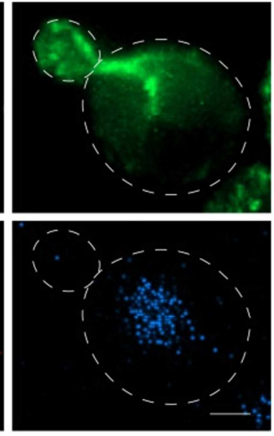

nls-tagRFP ${ }^{657}(3)$

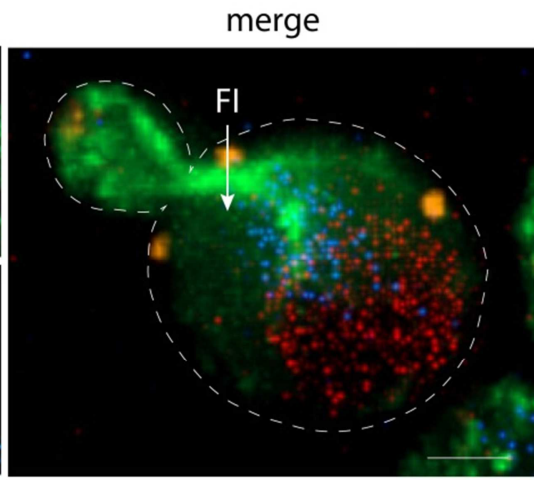

B
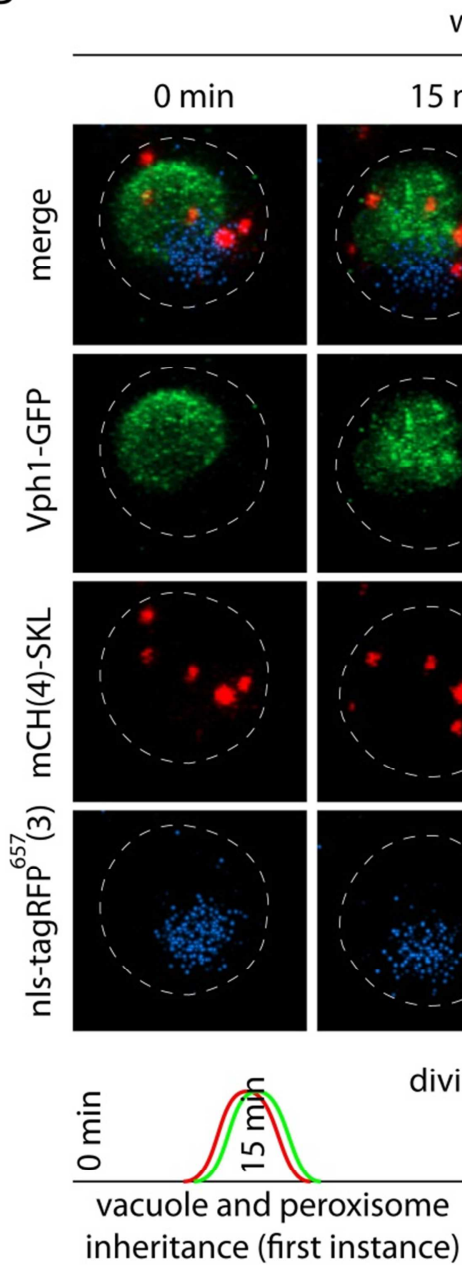

wt division
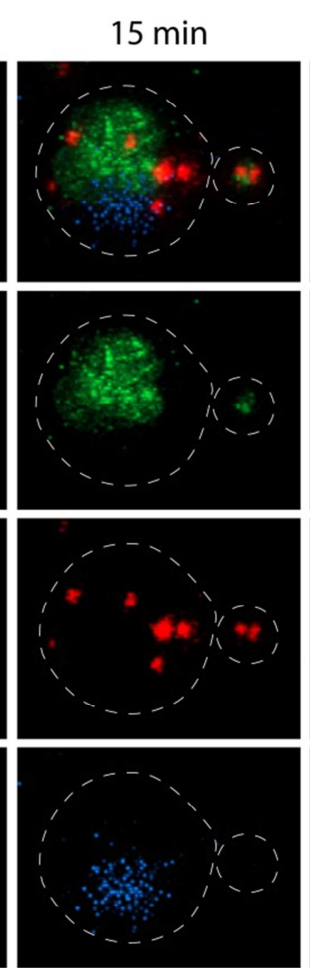

division timeline
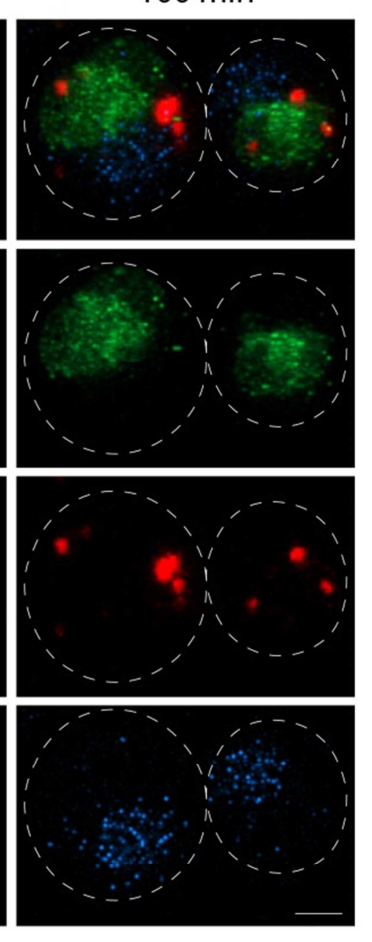

.

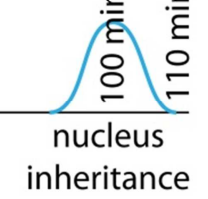

C

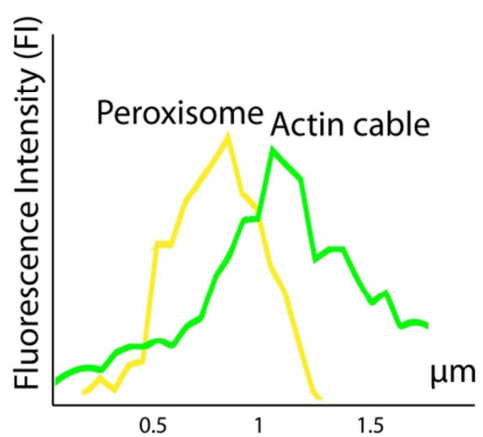

Ainp2 division
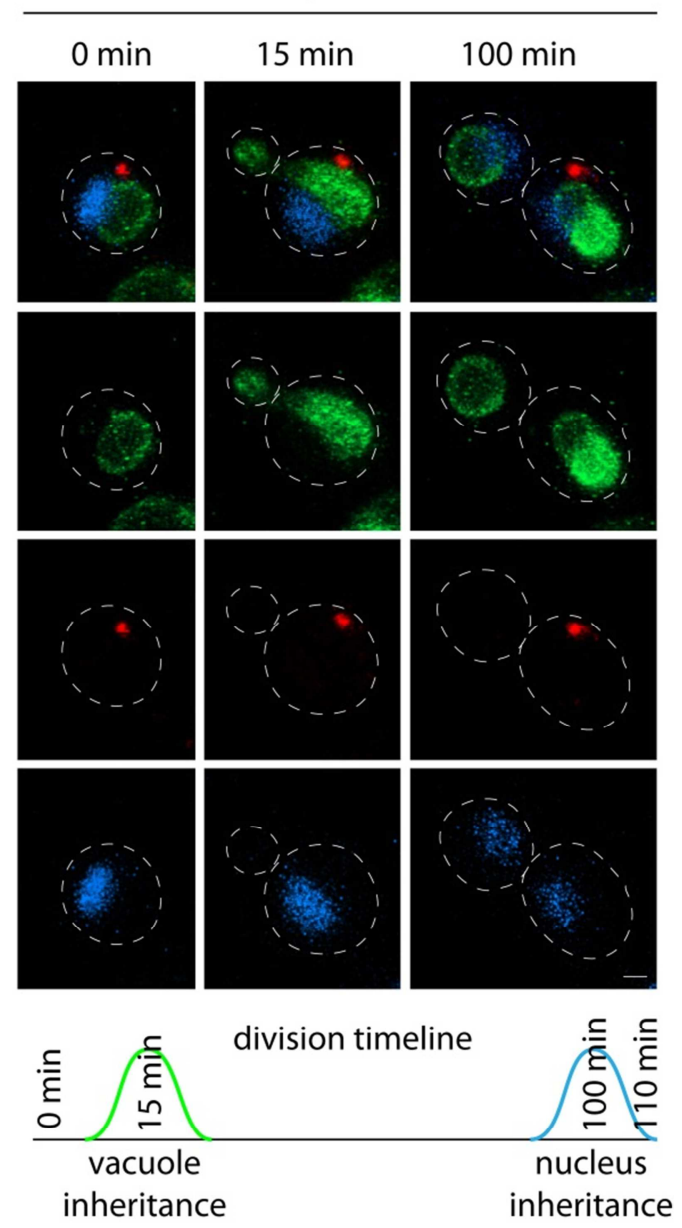

FIGURE 2: Proof of concept - Organelle inheritance order during division. (A) In vivo 4-color imaging of peroxisome (SKL signal C-terminally fused to 4 mCherry (mCH) on pDK-UT), actin (LifeAct fused to GFP on pDK-AT), nucleus (SV40 nls signal fused to 3 far-red fluorophores on pDK$\mathrm{HT}$ ), and vacuole (VPH1 endogenously tagged with $\mathrm{mBFP}$ ), the scale bar is $1 \mu \mathrm{m}$. The graph represents proximity of peroxisome to actin cable during division, Fluorescence Intensity (FI) was calculated along the $x$ axes (arrow on the merge image) for red (peroxisome) and green (actin) channels. (B) The timeline of organelle inheritance in the wild type (wt) strain, scale bar is $1 \mu \mathrm{m}$, time points represent the average ( $\mathrm{n}=30$ ) time of organelle inheritance from the start of division, strain carries SKL signal C-terminally fused to 4 mCherry (mCH) on pDK-UT module, SV40 nls signal fused to 3 far-red fluorophores on PDK-HT module, and VPH1 endogenously tagged with GFP. (C) The timeline of the organelle inheritance in the $\Delta$ inp2 strain, scale bar is $1 \mu \mathrm{m}$. 


\section{Application of pDK plasmids to multi-copy integration}

As proof of concept of exquisitely controlled inducible expression of a gene using multiple integrations we inserted GFP tagged VHL under inducible CUP1 promoter in 4 marker loci. The von Hippel Landau tumor suppressor (VHL) has often been used as a model substrate to study protein aggregation in yeast [23]. Carefully controlling expression levels of integrated proteins is essential to many studies. In the case of $\mathrm{VHL}$ and similar proteins, increased protein concentration results in gradual protein self-association with subsequent decrease in the monomer fraction in vitro. This leads to the formation of protein inclusions or aggregates. To test if there is a correlation of misfolded protein concentration and inclusion formation in vivo we introduced 1-4 copies of GFP-VHL under CUP1p (Figure 3A, B) in yeast. We scored inclusion formation as a function of concentration and temperature. Aggregation strongly correlates with an increase in temperature, $r$ (temperature) $=$ $0.76(p<0.05)$, but has no significant correlation with VHL concentration, $r$ (concentration) $=0.005(p=0.98)$. This could indicate a number of things. One interpretation of these data is that, regardless of the concentration, GFPVHL forms a small percentage of overall protein content in inclusions (because of the abundance of misfolded and unstructured proteins in yeast). Another possibility is that without significant heat shock the quality control system is able to degrade a spectrum of misfolded VHL concentrations, but at higher temperatures it becomes uniformly inhibited. Additional experiments could make similar titrations of proteasome and chaperone activity. Together, these data are compelling as proof-of-concept for multigene integrations.

\section{Bidirectional promoter sets}

To improve the time of strain construction, we built versatile bidirectional promoter sets, allowing integration of two inserts under different promoters at the same time. The promoters are positioned in the opposite orientation (Figure 1A). Previously described GAL1p/GAL1Op and 3 novel promoters: TEF1p-GPD1p, TEF1p-CUP1p, and TEF1p-DSE $4 p$
A

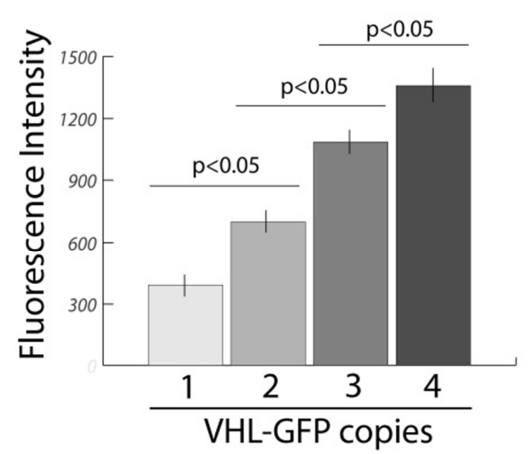

C

\begin{tabular}{|c|c|c|c|c|}
\hline & \multicolumn{4}{|c|}{ GFP-VHL } \\
\hline & 1 & 2 & 3 & 4 \\
\hline 느ํ & 0 & 0 & 0 & 0 \\
\hline ¿े & 0 & 0 & 0 & 0 \\
\hline $\begin{array}{l}u \\
\vdots \\
\grave{m}\end{array}$ & 0 & 0 & 1 & 2 \\
\hline$\stackrel{\text { ๖े }}{\stackrel{一}{*}}$ & 100 & 100 & 100 & 100 \\
\hline
\end{tabular}

$\mathrm{r}($ temperature $)=0.76(\mathrm{p}<0.01)$ $r($ concentration $)=0.005(p=0.98)$
B
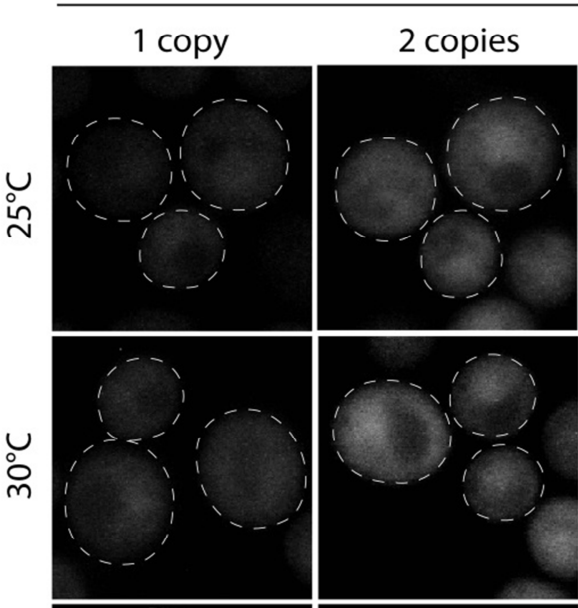

GFP-VHL
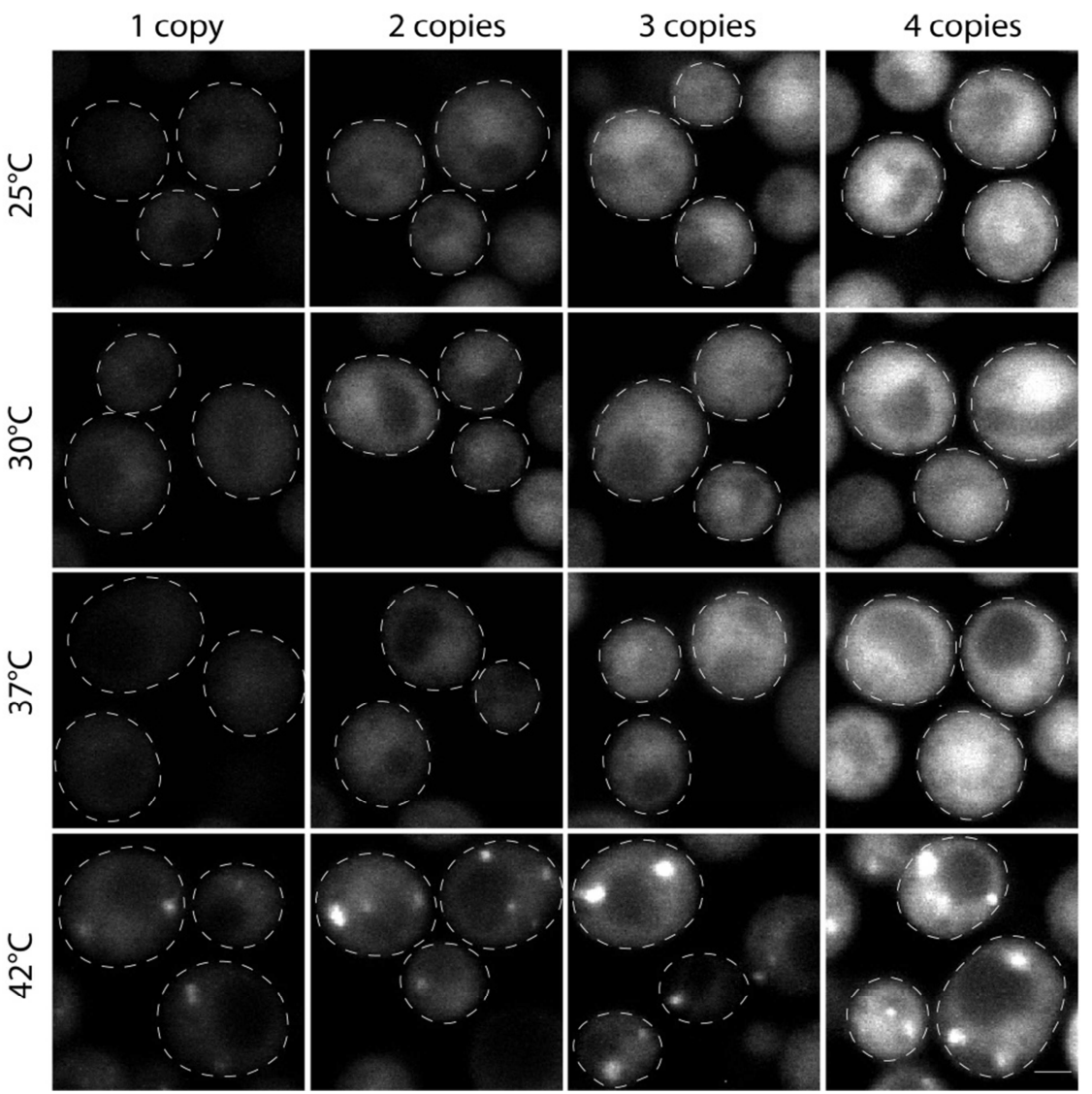

FIGURE 3: Proof of concept - Concentration does not correlate with inclusion formation. (A) Gradual increase in the amount of GFP-VHL according to integrated copies, Fluorescence Intensity was quantified in 30 single cells in the population, the average and standard errors are represented on the graph. (B) Confocal images of cells carrying 4 copies of GFP-VHL on PDK-AC, TC, UC, HC modules subjected to the range of temperatures for $1 \mathrm{~h}$, scale bar $1 \mu \mathrm{m}$. (C) Quantification of inclusion forming cells in the population according to the change in the temperature and the concentration, the numbers represent \% of the cells with inclusions $(n=300)$, correlation coefficient of aggregation and temperature ( $r$ (temperature)) and aggregation and concentration ( $r($ concentration $))$ is provided under the diagram. 
were introduced into pDK series. To validate promoter sets we constructed GFP/mCherry reporters and visualized the cells during the log phase (Figure 4). Bidirectional promoters allow controlled inducible, semi-inducible, constitutive and daughter-specific expression (Figure 4A-D). Not only do they facilitate strain construction, bidirectional sets can also be used for cell sorting (daughter/mother cells), study- ing aging, or screening yeast genome collections [24, 25].

In summary, the pDK vector series allows for efficient multiple integrations and thus is a useful tool for multicolor imaging, metabolic engineering, controlled expression of genes of interest, and stable yeast strain production. We therefore hope that $\mathrm{pDK}$ vectors will be a useful tool for the yeast community.
A
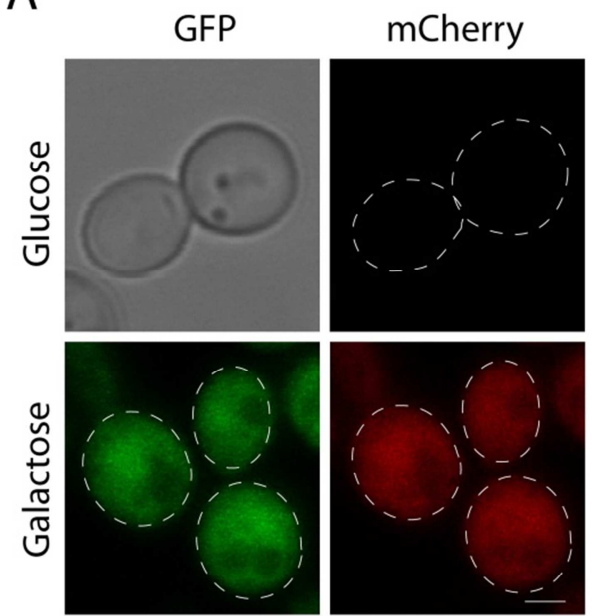

\begin{tabular}{|l|l|l|l|l|l}
\hline ADHt & GFP & GAL10p & GAL1p & mCherry & CYCt
\end{tabular}

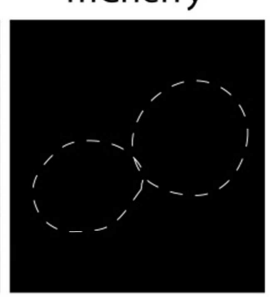

B

D

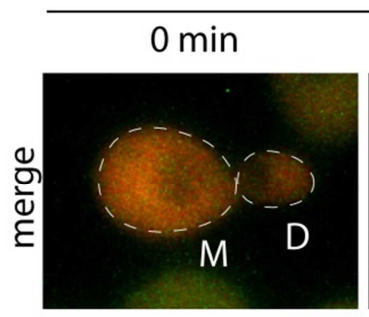

division timeline
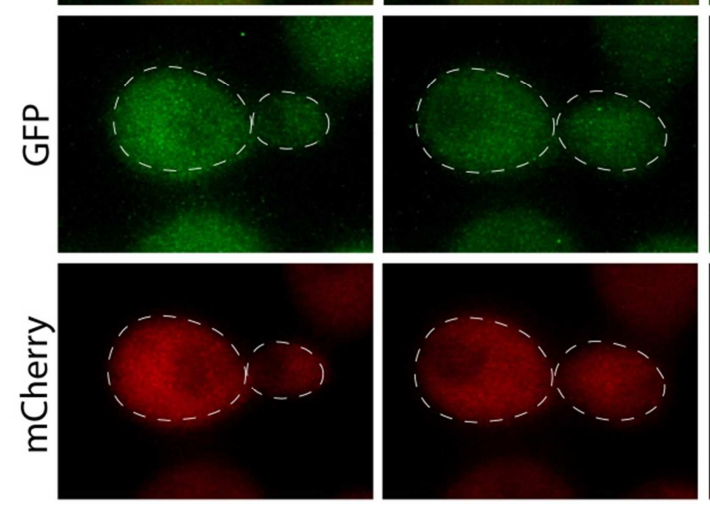

\begin{tabular}{l|l|l|l|}
\hline ADHt & GFP & DSE4p & TEF1P \\
\hline
\end{tabular}
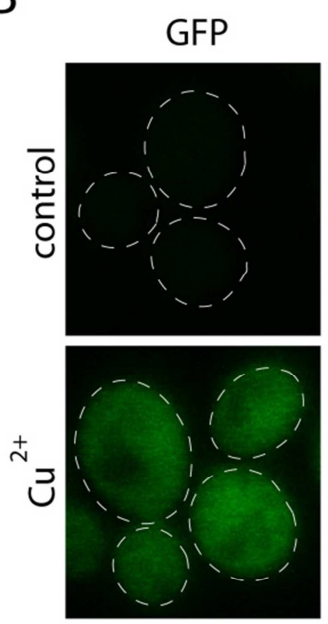

mCherry
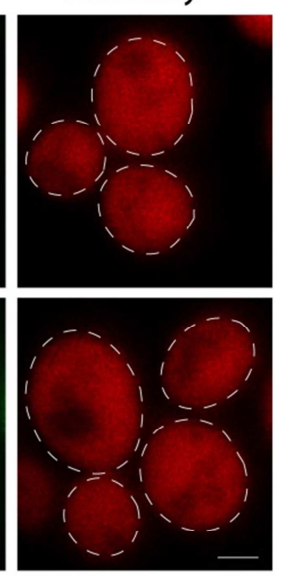

\begin{tabular}{l|l|l|l|l|l}
\hline ADHt & GFP & CUP1p & TEF1p & mCherry & CYCt
\end{tabular}
C

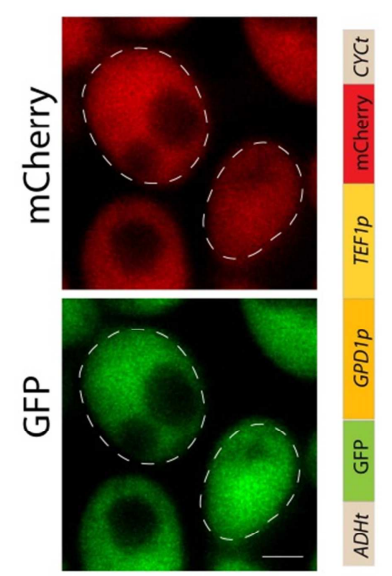

$\mathrm{E}$

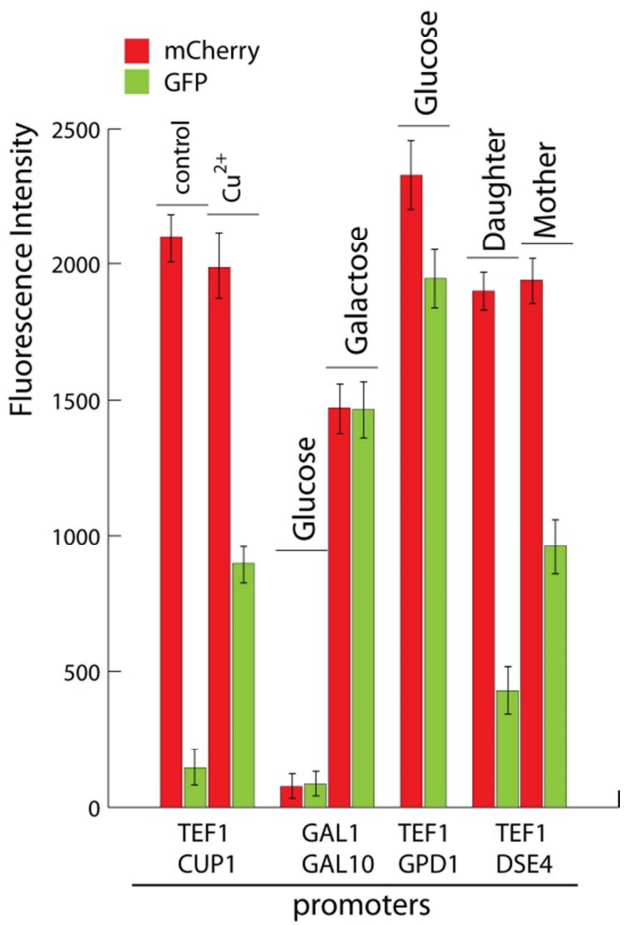

FIGURE 4: Bidirectional promoter reporters. (A) Galactose inducible bidirectional promoter, cells carrying pDK-HGG-GFP-mCherry were induced with galactose or grown on glucose for 6 hours, scale bar $1 \mu \mathrm{m}$. (B) Constitutive-inducible promoter, cells carrying pDK-HTC-GFP-mCherry were grown with and without (control) copper2+ for 4 hours, scale bar $1 \mu \mathrm{m}$. (C) Constitutive bidirectional promoter, cells carrying pDK-HTG-GFPmCherry were grown on glucose containing medium to middle log phase, scale bar $1 \mu \mathrm{m}$. (D) Daughter-specific-constitutive bidirectional promoter, cells carrying pDK-HTD-GFP-mCherry module were grown on glucose containing medium on the microscope, frames are shown (0 min, $60 \mathrm{~min}, 100 \mathrm{~min}$ ). (E) Comparison of fluorescence intensity of bidirectional reporters, the graph shows the average of fluorescence intensity of 20 cells and standard error. 


\section{MATERIALS AND METHODS}

\section{Strains and media}

We used standard conditions for culturing yeast and bacterial cells [26]. Yeast were grown in the selective medium $(1.7 \mathrm{~g} / \mathrm{l}$ yeast nitrogen base without amino acids and ammonium sulfate (Difco Laboratories), $5 \mathrm{~g} / \mathrm{l}$ ammonium sulphate, $0.77 \mathrm{~g} / \mathrm{l}$ complete, $2 \mathrm{~g} / \mathrm{l}$ amino acids supplement powder mix [27], 20 $\mathrm{g} / \mathrm{l}$ glucose, and $20 \mathrm{~g} / \mathrm{l}$ agar for the solid medium) or rich medium (20 g/l Peptone, $10 \mathrm{~g} / \mathrm{l}$ yeast extract, $20 \mathrm{~g} / \mathrm{l}$ glucose, and $20 \mathrm{~g} / \mathrm{l}$ agar for the solid medium). Galactose induction was performed on selective medium supplemented with $20 \mathrm{~g} / \mathrm{l}$ galactose instead of glucose for 6 hours. CUP1p induction was performed by addition of $50 \mu \mathrm{M}$ Cupric Sulphate to the selective medium and growing cells for 4 hours. Plasmids were constructed using Escherichia coli strain $\mathrm{DH} 5 \alpha$. Yeast strain W303-1B (MATa leu2-3,112 trp1-1 can1-100 ura3-1 ade2-1 his3-11,15) was used for the experiments.

\section{Plasmid construction}

pUC19 plasmid [28] was used as a backbone for pDK vectors. Primers used in this study are summarized in supplemental tables: 1 - primers used for yeast integration, 2 - primers used for construction of plasmids. Markers loci (including promoters and terminators) were split in the following parts: HIS3 (after $286 \mathrm{bp}$ of the ORF), URA3 (after $420 \mathrm{bp}$ of the ORF), TRP1 (after $224 \mathrm{bp}$ of the ORF), ADE2 (after $462 \mathrm{bp}$ of the ORF). Split fragments were amplified with: HIS3F1/R1 and HIS3F2/R2, URA3F1/R1 and URA3F2/R2, TRP1F1/R1 and TRP1F2/R2, ADE2F1/R1 and ADE2F2/R2, and cloned into pUC19's Pcil/HindIII and Pfol/EcoRl, EcoRl/Pfol and Pcil/HindIII, HindIII/Pcil and Pfol/EcoRI, HindIII/Pcil and Pfol/EcoRI respectively, resulting in the plasmids $\mathrm{PDK}-\mathrm{HH}, \mathrm{pDK}-\mathrm{UU}, \mathrm{PDK}-\mathrm{TT}$, and pDK-AA. Promoters and terminators (TEF1p, CUP1p, TEF1t) were amplified with: TEF1PF/R, CUP1PF/R, TEF1TF/R primers and cloned into pUC19's Ndel/EcoRI, Ndel/EcoRI, and Sall/Hindlll sites, respectively, resulting in pUC19-TEF1p-TEF1t and pUC19-CUP1p-TEF1t plasmids. TEF1p-TEF1t, CUP1p-TEF1t and GAL1/10p-ADH1/CYC1t modules were amplified from previously constructed pUC19-TEF1p-TEF1t and pUC19CUP1p-TEF1t, and pESC-URA (Agilent) vectors using the primers: CUPTEFHHF, CUPTEFUUF, CUPTEFTTF, CUPTEFAAF and CUPTEFR for TEF1p-TEF1 and, CUP1p-TEF1t modules, and GALHHF, GALUUF, GALTTF, GALAAF and GALR for GAL1/10p$A D H 1 / C Y C 1 t$ module, and cloned using Gibson Assembly mix (NEB) into pDK-HH, pDK-UU, pDK-TT, and pDK-AA vectors linearized with $\mathrm{EcoRI} / \mathrm{Sall}$ restriction enzymes resulting in $12 \mathrm{pDK}$ plasmids. Plasmid names contain the first letter of the marker followed by the first letter/s of the promoter/s (Table 1). TEF1 promoter was amplified with TEFbF/R primers and cloned into pESC-URA Agel/BamHI sites resulting in the pESC-TEFp plasmid. CUP1, DSE4, and GPD1 promoters were amplified with $\mathrm{CUPbF} / \mathrm{R}, \mathrm{DSEbF} / \mathrm{R}$, and GPDbF/R primers and cloned into the pESC-TEFp vector into Agel/ECoRI sites resulting in pESC-TG, TC and TD plasmids carrying bidirectional promoters. Bidirectional promoters were subcloned using BamHI/EcoRI into pDKHG/UG/TG/AG, resulting in 12 bidirectional promoter sets (Table 1).

The nuclear cellular marker plasmid was constructed by cloning the SV40 nuclear localization signal fused to (tagRFP657)4 [29] into pDK-HT vector EcoRI/Smal sites. The peroxisome marker was constructed by cloning the (mCherry)4SKL sequence into pDK-UT plasmid. Actin was visualized via the LifeAct fragment fused to GFP [16], LifeAct-GFP was cloned into the PDK-AT vector. VHL plasmids were constructed by cloning the GFP-VHL sequence into pDK-HC, pDK-UC, pDK$A C$, and pDK-TC vectors. pCfB2513 was a gift from Irina Borodina (Addgene plasmid \# 67543).

For endogenous tagging we modified the pKT127 [30]

TABLE 2. Strains used in this study.

\begin{tabular}{|c|c|}
\hline Strain & Genotype \\
\hline BY4741 & MATa his $3 \Delta 0$ leu2 $\Delta 0$ met $15 \Delta 0$ ura $3 \Delta 0$ \\
\hline W303 $\alpha$ & MATa leu2-3,112 trp1-1 can1-100 ura3-1 ade2-1 his3-11,15 \\
\hline yDK - inp2, nuc, pex, vac & $\begin{array}{l}\text { W303 } \alpha \text { inp2 } \triangle:: K a n M X \text { his3-11,15::nls-(tagRFP657) })_{3}: \text { HIS3 ura3-1:(mCherry) } 3^{-} \\
\text {SKL::URA3 VPH1-GFP-HPH }\end{array}$ \\
\hline yDK - nuc, pex, vac & $\begin{array}{l}\left.\text { W303 } \alpha \text { his3-11,15::nls-(tafRFP657) })_{3}: \text { HIS3 ura3-1:(mCherry) }\right)_{3} \text {-SKL::URA3 VPH1-GFP- } \\
\text { HPH }\end{array}$ \\
\hline yDK - nuc, pex, la, vac & $\begin{array}{l}\text { W303 } \alpha \text { his3-11,15::n/s-(tafRFP657) }::: H I S 3 \text { ura3-1:(mCherry) }{ }_{3} \text {-SKL::URA3 ade2- } \\
\text { 1::LifeAct-GFP::ADE2 VPH1-mBFP-HPH }\end{array}$ \\
\hline YDK - HHCTGV & W303 $\alpha$ his3-11,15::CUP1p-GFP-VHL-TEF1t::HIS3 \\
\hline YDK - HHCTGV, UUCTGV & $\begin{array}{l}\text { W303 } \alpha \text { his3-11,15::CUP1p-GFP-VHL-TEF1t::HIS3 ura3-1::CUP1p-GFP-VHL- } \\
\text { TEF1t::URA3 }\end{array}$ \\
\hline yDK - HHCTGV, UUCTGV, TTCTGV & $\begin{array}{l}\text { W303 } \alpha \text { his3-11,15::CUP1p-GFP-VHL-TEF1t::HIS3 ura3-1::CUP1p-GFP-VHL- } \\
\text { TEF1t::URA3 trp1-1::CUP1p-GFP-VHL-TEF1t::TRP1 }\end{array}$ \\
\hline yDK - HHCTGV, UUCTGV, TTCTGV, AACTGV & $\begin{array}{l}\text { W303 } \alpha \text { his3-11,15::CUP1p-GFP-VHL-TEF1t::HIS3 ura3-1::CUP1p-GFP-VHL- } \\
\text { TEF1t::URA3 trp1-1::CUP1p-GFP-VHL-TEF1t::TRP1 ade2-1::CUP1p-GFP-VHL- } \\
\text { TEF1t::ADE2 }\end{array}$ \\
\hline
\end{tabular}


plasmid by inserting the $\mathrm{mBFP}$ sequence or GFP sequence into $\mathrm{PaCl} / \mathrm{Ascl}$ sites and $\mathrm{HPH}$ marker sequence into $\mathrm{Bg} / \mathrm{ll} / \mathrm{Pmel}$ sites. GFP reporters were constructing by cloning GFP into $\mathrm{Sacl} / \mathrm{Xmal}$ sites of $\mathrm{pDK}-\mathrm{HT} / \mathrm{HC}$, and BamHI/Xmal sites of pDKHGG with primers GFPXR, GFPbF, GFPsF and subcloning the fragment containing the marker across different marker plasmids and bidirectional promoter ones. mCherry was amplified with primers $\mathrm{CHeF}$ and $\mathrm{CHnR}$ and cloned into the Ecol/Spel sites of pDK-HGG-GFP, and subcloned to pDK-HTC-GFP, pDKHTG-GFP, and pDK-HTD-GFP.

\section{Strain construction}

We introduced the INP2 deletion using a PCR based deletion strategy [31] and primers delINPF/R. Gene deletions were verified by PCR. Strains with integrative modules were constructed by transforming yeast with a PCR fragment obtained from a corresponding plasmid with a set of primers listed in Table 1. For comparison experiments pRS plasmids were linearized in the marker locus prior to integration, pRS303 and pRS306 with Pstl restriction enzyme, and pRS304 with Pm/l enzyme.

VPH1 was tagged using modified pKT127 plasmid and eVPHF/R primers.

\section{Protocol for yeast integration}

The fragment for genomic integration is generated via PCR with primers listed in Table 2 using the following parameters $\left(95^{\circ} \mathrm{C}-5^{\prime},\left[95^{\circ} \mathrm{C}-30^{\prime \prime}, 62^{\circ} \mathrm{C}-30^{\prime \prime}\right.\right.$ (increment $0.8^{\circ} \mathrm{C}$ per cycle), $72^{\circ} \mathrm{C}-\mathrm{X}$ min $\left(\mathrm{X}=\right.$ length of the fragment in kb)] 25 cycles, $72^{\circ} \mathrm{C}$ $\left.5^{\prime}\right)$, and high fidelity polymerase generating blunt-end products, e.g. KAPA HiFi DNA Polymerase (KAPA Biosystems). A PCR protocol with fixed primer binding time can also be used. Up to $2 \mu \mathrm{g}$ of PCR product is transformed using LiAc/PEG transformation [32] with modifications $-50 \mu \mathrm{l}$ of DMSO is added prior to heat shock, heat shock time is reduced to 15 min at $42^{\circ} \mathrm{C}$. 20 random clones carrying integrative modules were verified by PCR.

\section{Stability of integration}

Yeast strains were grown in rich medium in three replicates. The culture was diluted $1 / 100$ every 24 hours for 10 days. The culture was analyzed by reporter fluorescence on the first and tenth day.

\section{Confocal Microscopy}

For imaging yeast cells were grown to mid-log phase and seeded on concanavalin A (Sigma) coated 4-well microscope plates (IBIDI). For induction galactose rich media (division experiments, Figure 2) or minimal media with $50 \mu \mathrm{m} \mathrm{Cu}{ }^{2+}$ (Figure 3) were used. Copper induced cells were grown in $25^{\circ} \mathrm{C}$ to

\section{REFERENCES}

1. Sikorski RS, Hieter P (1989). A System of Shuttle Vectors and Yeast Host Strains Designed for Efficient Manipulation of DNA in Saccharomyces-Cerevisiae. Genetics 122(1): 19-27.

2. Fang F, Salmon K, Shen MW, Aeling KA, Ito E, Irwin B, Tran UP, Hatfield GW, Da Silva NA, Sandmeyer $S$ (2011). A vector set for systematic metabolic engineering in Saccharomyces cerevisiae. Yeast 28(2): 123-136. mid-log phase and then incubated for $1 \mathrm{~h}$ at indicated temperatures $\left(30,37,42^{\circ} \mathrm{C}\right)$. Confocal $3 \mathrm{D}$ images and movies were acquired using a dual point-scanning Nikon A1R-si microscope equipped with a PInano Piezo stage (MCL), using a $60 \mathrm{x}$ PlanApo VC oil objective NA 1.40. Movies were acquired in resonant-scanning mode. Image processing was performed using NIS-Elements software.

\section{Statistics}

The experiments were repeated at least 3 times. Multiple correlation coefficients were calculated for 3 variables (aggregation, temperature, concentration), with subsequent regression analysis to determine $p$-values. Standard comparisons were performed using t-test.

\section{ACKNOWLEDGEMENTS}

We thank members of the Jerusalem Brain Community for their support. This work was supported by the European Research Council under the European Union's Seventh Framework Programme (FP/2007-2013)/ERC-StG2013 337713 DarkSide starting grant, as well as an Israel Science Foundation Grant ISF 843/11; a German Israel Foundation Grant GIFI-1201-242.13/2012; a Niedersachsen-Israel Research Program grant, an Abisch-Frenkel Foundation grant, and a joint Israel-Italy cooperation grant from the Israeli Ministry of Science, Technology, and Space. TA was funded by a Jerusalem Brain Community Doctoral Fellowship and by the Alexander Grass Center for Bioengineering.

\section{SUPPLEMENTAL MATERIAL}

All supplemental data for this article are available online at www.microbialcell.com.

\section{CONFLICT OF INTEREST}

The authors declare no conflicts of interest.

\section{COPYRIGHT}

(C) 2017 Amen and Kaganovich. This is an open-access article released under the terms of the Creative Commons Attribution (CC BY) license, which allows the unrestricted use, distribution, and reproduction in any medium, provided the original author and source are acknowledged.

Please cite this article as: Triana Amen and Daniel Kaganovich (2017). Integrative modules for efficient genome engineering in yeast. Microbial Cell 4(6):182-190. doi: 10.15698/mic2017.06.576

3. Alberti S, Gitler AD, Lindquist S (2007). A suite of Gateway (R) cloning vectors for high-throughput genetic analysis in Saccharomyces cerevisiae. Yeast 24(10): 913-919.

4. Ronda C, Maury J, Jakociunas T, Jacobsen SAB, Germann SM, Harrison SJ, Borodina I, Keasling JD, Jensen MK, Nielsen AT (2015). CrEdit: CRISPR mediated multi-loci gene integration in Saccharomyces cerevisiae. Microb Cell Fact 14:97. 
5. Agmon N, Mitchell LA, Cai YZ, Ikushima S, Chuang J, Zheng A, Choi WJ, Martin JA, Caravelli K, Stracquadanio G, Boeke JD (2015). Yeast Golden Gate (yGG) for the Efficient Assembly of S-cerevisiae Transcription Units. Acs Synth Biol 4(7): 853-859.

6. Stovicek V, Borja GM, Forster J, Borodina I (2015). EasyClone 2.0: expanded toolkit of integrative vectors for stable gene expression in industrial Saccharomyces cerevisiae strains. J Ind Microbiol Biot 42(11): 1519-1531.

7. Leite FCB, dos Anjos RSG, Basilio ACM, Leal GFC, Simoes DA, de Morais MA (2013). Construction of integrative plasmids suitable for genetic modification of industrial strains of Saccharomyces cerevisiae. Plasmid 69(1): 114-117.

8. Kung SH, Retchless AC, Kwan JY, Almeida RPP (2013). Effects of DNA Size on Transformation and Recombination Efficiencies in Xylella fastidiosa. Appl Environ Microb 79(5): 1712-1717.

9. Jensen NB, Strucko T, Kildegaard KR, David F, Maury J, Mortensen UH, Forster J, Nielsen J, Borodina I (2014). EasyClone: method for iterative chromosomal integration of multiple genes in Saccharomyces cerevisiae. Fems Yeast Res 14(2): 238-248

10. Jahn M, Vorpahl C, Hubschmann T, Harms H, Muller S (2016). Copy number variability of expression plasmids determined by cell sorting and Droplet Digital PCR. Microb Cell Fact 15(1): 211.

11. Sikorski RS, Hieter $P$ (1989). A system of shuttle vectors and yeast host strains designed for efficient manipulation of DNA in Saccharomyces cerevisiae. Genetics 122(1): 19-27.

12. Horwitz AA, Walter JM, Schubert MG, Kung SH, Hawkins K, Platt DM, Hernday AD, Mahatdejkul-Meadows T, Szeto W, Chandran SS, Newman JD (2015). Efficient Multiplexed Integration of Synergistic Alleles and Metabolic Pathways in Yeasts via CRISPR-Cas. Cell Syst 1(1): 88-96.

13. Shi SB, Liang YY, Zhang MZM, Ang EL, Zhao HM (2016). A highly efficient single-step, markerless strategy for multi-copy chromosomal integration of large biochemical pathways in Saccharomyces cerevisiae. Metab Eng 33:19-27.

14. Kuijpers NGA, Chroumpi S, Vos T, Solis-Escalante D, Bosman L, Pronk JT, Daran JM, Daran-Lapujade P (2013). One-step assembly and targeted integration of multigene constructs assisted by the I-Scel meganuclease in Saccharomyces cerevisiae. Fems Yeast Res 13(8): 769-781.

15. Taxis C, Knop M (2006). System of centromeric, episomal, and integrative vectors based on drug resistance markers for Saccharomyces cerevisiae. Biotechniques 40(1): 73-78.

16. Cho SW, Kim S, Kim Y, Kweon J, Kim HS, Bae S, Kim JS (2013). Analysis of off-target effects of CRISPR/Cas-derived RNA-guided endonucleases and nickases. Genome Research 24(1): 132-141.

17. Riedl J, Crevenna AH, Kessenbrock K, Yu JH, Neukirchen D, Bista $M$ Bradke F, Jenne D, Holak TA, Werb Z, Sixt M, Wedlich-Soldner R (2008). Lifeact: a versatile marker to visualize F-actin. Nat Methods 5(7): 605607.
18. Spokoini R, Shamir M, Keness A, Kaganovich D (2013). 4D Imaging of Protein Aggregation in Live Cells. Jove-J Vis Exp (74).

19. Hoepfner $D$, van den Berg M, Philippsen P, Tabak HF, Hettema EH (2001). A role for $\mathrm{Vps} 1 \mathrm{p}$, actin, and the Myo2p motor in peroxisome abundance and inheritance in Saccharomyces cerevisiae. J Cell Biol 155(6): 979-990.

20. Fagarasanu A, Fagarasanu M, Eitzen GA, Aitchison JD, Rachubinski RA (2006). The peroxisomal membrane protein Inp2p is the peroxisome-specific receptor for the myosin $\mathrm{V}$ motor Myo2p of Saccharomyces cerevisiae. Dev Cell 10(5): 587-600.

21. Saraya R, Cepinska MN, Kiel JAKW, Veenhuis M, van der Klei IJ (2010). A conserved function for Inp2 in peroxisome inheritance. BbaMol Cell Res 1803(5): 617-622.

22. Knoblach B, Rachubinski RA (2015). Sharing the cell's bounty organelle inheritance in yeast. J Cell Sci 128(4): 621-630.

23. Beach DL, Thibodeaux J, Maddox P, Yeh E, Bloom K (2000). The role of the proteins Kar9 and Myo2 in orienting the mitotic spindle of budding yeast. Curr Biol 10(23): 1497-1506.

24. Brock KP, Abraham AC, Amen T, Kaganovich D, England JL (2015). Structural Basis for Modulation of Quality Control Fate in a Marginally Stable Protein. Structure 23(7): 1169-1178.

25. Li A, Liu Z, Li Q, Yu L, Wang D, Deng X (2008). Construction and characterization of bidirectional expression vectors inSaccharomyces cerevisiae. Fems Yeast Res 8(1): 6-9.

26. Afonso B, Silver PA, Ajo-Franklin CM (2010). A synthetic circuit for selectively arresting daughter cells to create aging populations. Nucleic Acids Research 38(8): 2727-2735.

27. Sherman F (2002). Getting started with yeast. Method Enzymol 350:3-41.

28. Baryshnikova A, Costanzo M, Dixon S, Vizeacoumar FJ, Myers CL, Andrews B, Boone C (2010). Synthetic Genetic Array (SGA) Analysis in Saccharomyces cerevisiae and Schizosaccharomyces pombe. 470:145179.

29. Yanischperron C, Vieira J, Messing J (1985). Improved M13 Phage Cloning Vectors and Host Strains - Nucleotide-Sequences of the M13mp18 and Puc19 Vectors. Gene 33(1): 103-119.

30. Morozova KS, Piatkevich KD, Gould TJ, Zhang JH, Bewersdorf J, Verkhusha VV (2010). Far-Red Fluorescent Protein Excitable with Red Lasers for Flow Cytometry and Superresolution STED Nanoscopy. Biophys J 99(2): L13-L15.

31. Sheff MA, Thorn KS (2004). Optimized cassettes for fluorescent protein tagging in Saccharomyces cerevisiae. Yeast 21(8): 661-670.

32. Baudin A, Ozierkalogeropoulos O, Denouel A, Lacroute F, Cullin C (1993). A Simple and Efficient Method for Direct Gene Deletion in Saccharomyces-Cerevisiae. Nucleic Acids Res 21(14): 3329-3330.

33. Gietz RD, Schiestl RH, Willems AR, Woods RA (1995). Studies on the Transformation of Intact Yeast-Cells by the Liac/S-DNA/Peg Procedure. Yeast 11(4): 355-360. 